\title{
On the Access Pricing Issues of Wireless Mesh Networks
}

\author{
Ray K. Lam* Dah-Ming Chiu ${ }^{\dagger} \quad$ John C.S. Lui*
}

\begin{abstract}
This paper studies the use of pricing as an incentive mechanism to encourage private, self-interested nodes to participate in a public wireless mesh network and cooperate in the packet forwarding service. Our focus is on the "economic behavior" of the network nodes-the pricing and purchasing strategies of the access point, wireless relaying nodes, and clients. We use a "game theoretic approach" to analyze their interactions from one-hop to multihop network and when the network has an unlimited or limited channel capacity. We show that the access point and relaying wireless nodes will adopt a simple, yet optimal, fixed-rate pricing strategy in a multi-hop network with an unlimited capacity. Yet, the fixed-rate pricing strategy fails to be optimal in the limited capacity case. To this end, we focus on the access point adopting a more practical "fixedrate, non-interrupted service" model and propose an algorithm based on the Markovian decision theory to devise the optimal pricing strategy.
\end{abstract}

\section{Introduction}

The growing interest of wireless mesh network technology together with the growing popularity of wireless network devices at homes, offices and public places induce a vision that when wireless mesh network is deployed in the public, we would have nearly ubiquitous wireless coverage in urban areas. Yet an important question left unanswered is why private access points and wireless nodes would participate in a public mesh network and act in a cooperative manner. Connectivity in a mesh network relies on nodes forwarding packets for each other, but relaying packets incurs costs to a node, in terms of reduced bandwidth, energy consumption, potential security risks, etc. In community mesh networks, cooperation can be assumed, but in the public domain, nodes in the network will be self-interested, or

\footnotetext{
*Department of Computer Science \& Engineering, The Chinese University of Hong Kong, Shatin, NT, Hong Kong; $\{$ klam,cslui\}@cse.cuhk.edu.hk.

$\dagger^{\dagger}$ Department of Information Engineering, The Chinese University of Hong Kong, Shatin, NT, Hong Kong; dmchiu@ie.cuhk.edu.hk.
}

economically rational. Without incentives, we should not assume these nodes to cooperate.

In this work, we study the use of pricing as a mechanism to encourage participation and cooperation in a public mesh network. As objectives of most nodes would be to access the Internet, we take "Internet access" as a service, and hence access points are the service sellers. Any downstream wireless nodes may purchase this service, for her own consumption, or for reselling it to nodes further downstream. Transactions involved must be on a per-access basis, using technology such as the PayWord micro-payment scheme [11,9], which minimizes the transaction overhead. Monthly prepayment scheme or the like is impossible as nodes here concerned are not reliable to provide consistent service in the long run. By this access provision business, participating nodes generate revenue to compensate their costs for packet forwarding. We investigate the economic behavior of different nodes in the network. In particular, we seek to answer these questions:

- How will the access point and different wireless relaying nodes set their prices for the service?

- Will their optimal pricing schemes be complicated, such as the access point charging a floating rate with time, which may discourage clients for the service?

- Does the pricing mechanism requires third-party supervision?

We believe answers to these questions will shed light into the deployment of public wireless mesh networks.

Our analysis adopts a game theoretic approach to find out the strategies that the access points, relaying wireless nodes, and clients will play throughout the bargaining process at equilibrium. We focus on mesh networks in which there is a single access point having the Internet connectivity, and every wireless client has a single path toward this access point. Figure 1 shows three examples of such a tree-like network. We differentiate two cases in this setting: (1) the wireless network and the access point's wired uplink to the Internet have an unlimited capacity (or the capacity is sufficiently large to satisfy all demands); (2) the network has a limited capacity. In each case, we first look at a one-hop network depicted in Figure 1(a), in which all 


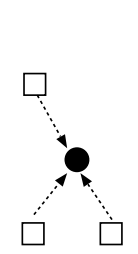

(a) One-hop wireless network

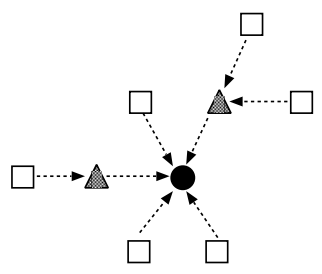

(b) Two-hop wireless network

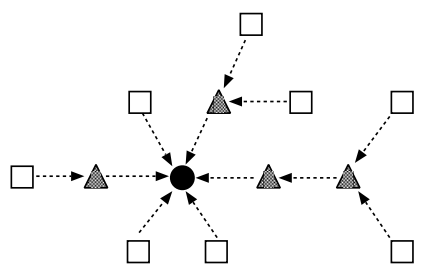

(c) Multi-hop wireless network
Access point

$\triangle$ Relaying wireless node

$\square$ Client

\section{Figure 1. Various wireless mesh networks analyzed in this paper}

clients can reach the access point directly; then we extend it to the multi-hop case as in Figure 1(c), in which clients have to route through numerous relaying wireless nodes, or resellers, to the access point, in order to receive the Internet access service. Note that the one-hop case and two-hop case (Figure 1(b)) under the unlimited capacity assumption are first studied in the seminal work by Musacchio and Walrand [10]. Studying pricing under the unlimited capacity assumption is worthwhile, as it provides asymptotic results as the wired and wireless network capacity go abundant, which can be foreseen due to technology maturity. The limited capacity model offers a more realistic investigation, and we expect the access point to play a rather different strategy when she can only sell her service to a limited number of clients. Adopting a tree-like network model simplifies the problem and provides us the basic pricing structure in wireless mesh networks.

The contributions of this work are summarized as follows. First, we generalize the model in [10] and show that it is only a special case when the network has an unlimited capacity, or equivalently, has an adequate supply of bandwidth to meet all demands from clients. The elegant results in the unlimited capacity model - the access point and resellers charging a "fixed rate" at all time-no longer apply in the limited capacity case. Secondly, we extend the twohop case of the unlimited capacity model in [10] to study multi-hop networks in general. Third, in view of the fixedrate pricing strategy being non-optimal when the network has a limited capacity, we propose a more practical charging policy, the "fixed-rate, non-interrupted service", for wireless Internet access. Under this policy, we use the policyiteration method from the Markovian decision theory to devise the optimal pricing strategy of the access point. The algorithm is made applicable to both the one-hop case and the multi-hop case of the limited capacity model.

The balance of this paper is as follows. In Section 2 we discuss background and related results in [10], forming the basis of our work. In Section 3 we extend the unlimited capacity model to the multi-hop case by proposing an equi- librium strategy profile. In Section 4 we investigate the limited capacity model, showing the previous equilibrium no longer holds, then we present the fixed-rate, non-interrupted service model and devise the optimal pricing strategy of the access point using the Markovian decision theory. We finish the section with an analysis of the multi-hop case. Section 5 concludes.

\section{Related Work and Background}

While pricing in computer network has been receiving much attention from the community for years, only recently study has been conducted on wireless network, which has different characteristics from traditional wired network. For wired network, researchers mainly focus on pricing as a mechanism for admission and congestion control [8,5], while our work focuses on pricing as an incentive system and the related economic issues of wireless networks. Among the published results focusing on wireless network, the most related ones to our work are $[10,2,1,12]$. In [10], authors only study the pricing issue under the unlimited capacity assumption. In [2], authors study the pricing of wireless network but with the emphasis on searching for a strategy-proof pricing mechanism. It provides only limited analysis apart from simple fixed-rate pricing. In [1], authors adopt a demand-and-supply framework to analyze pricing dynamics in two-hop networks with one or more service providers. In contrast, our work focuses on monopoly markets in multi-hop networks and studies the strategic behavior of the access point, relaying nodes and clients which target at payoff maximization based on their underlying utility functions. In [12], authors investigate pricing in general wireless communication network and address performance issues. Our work places the problem scenario in a wireless mesh network and studies pricing as an incentive mechanism to stimulate participation and cooperation.

The seminal work by Musacchio and Walrand [10] presents the economic behavior of wireless nodes under a specific network topology. In particular, they study "one- 
hop" and "two-hop" wireless networks using a game theoretic approach, and prove that "fixed-rate pricing" is optimal to the access point, given that clients have the socalled "web browsing" utility function. Web browsing utility function models, for a client browsing the web, her utility of having Internet access-the utility grows proportionally with the time she gains access initially, and saturates when she no longer intends to browse. Note that the analysis adopted and the results proven are only valid under a strong assumption: the network has an unlimited capacity, i.e. the channel capacity of the wireless network is unlimited and the access point has an unlimited uplink bandwidth to the Internet, or the access point provides no bandwidth guarantee to clients, while clients value the connection service without considering the available bandwidth. This assumption allows the access point to admit infinitely many clients; the admission of one client has no influence on the admission of others. Thus, the access point's total profit can be maximized by separately maximizing her gain in each interaction with a client. In the one-hop case, a two-player game between the access point and a single client abstracts all details of the aggregated system; while in the two-hop case, a three-player game among the access point, a single relaying node, and a single client will do. Our work relaxes the unlimited capacity assumption and shows that fixed-rate pricing is no longer optimal to the access point. The model adopted by [10] is hereafter termed unlimited capacity model. In the following, we first present the related results, which serve as the basis of our work.

\subsection{Unlimited Capacity Model-One-hop Case}

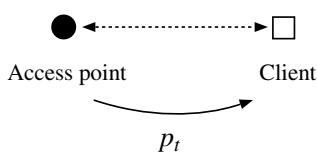

\section{Figure 2. Game modeling of the one-hop case with a slot price $p_{t}$ charged by the access point}

The one-hop case of the unlimited capacity model describes a wireless network where all clients can reach the access point directly (i.e., without the need of packet forwarding by other nodes). The dynamics among the access point and the numerous clients is captured using a twoplayer game between the access point and a single client as shown in Figure 2. Time is divided into discrete slots. At the beginning of each time slot $t$, the client requests for connection service over the slot and the access point replies with a slot price $p_{t}$. The client chooses to accept the price and connect to the access point, or to reject and leave. The game ends once the client rejects a slot price, and the number of time slots the client connects is denoted by $T$. The client has a web browsing utility function:

$$
F(T, \tau)=U \cdot \min (T, \tau),
$$

where $\tau$ is a discrete random variable representing the number of time slots the client intends to connect and browse the web, and $U$ is a continuous random variable representing the client's utility of gaining Internet access in one time slot. The client knows her values of $U$ and $\tau$, while the access point's prior knowledge of them includes only their probability distributions, obtained for example from market survey. ${ }^{1}$ Upon the end of the game, the client has a net payoff of $F(T, \tau)-\sum_{t=1}^{T} p_{t}$, while the access point has a profit of $\sum_{t=1}^{T} p_{t}$. Authors in [10] prove that the following strategy profile is a perfect Bayesian equilibrium (PBE) [3]:

- The client connects or remains connected in slot $t$ iff $t \leq \tau$ and $p_{t} \leq U$

- The access point charges a non-decreasing price sequence $\left\{p_{t}\right\}$ such that

$$
p_{t} \in \arg \max _{p} p P(U \geq p)
$$

There are two points to be noted here. First, the client's strategy is named the "myopic strategy", for its sole dependence on the immediate slot price. Second, it is often the case that the access point charges a "constant", or fixed price sequence, since the expression $p P(U \geq p)$ is maximized by a single price $p^{*}$ for most distributions of $U$, and the price $p^{*}$ does not vary over time slots.

\subsection{Unlimited Capacity Model-Two-hop Case

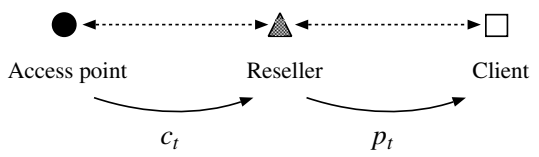

Figure 3. Game modeling of the two-hop case with a slot price $c_{t}$ charged by the access point, and a slot price $p_{t}$ charged by the reseller

The two-hop case describes the situation when a client is incapable of reaching the access point directly, but has to

\footnotetext{
${ }^{1}$ It will be trivial if $U$ is deterministic to the access point. The access point will charge the client a slot price of $p_{t}=U$, given that the client will choose to pay and connect rather than to reject the price, while both yield her zero payoff.
} 
route her traffic through an intermediate wireless node, referred as the reseller. The game now involves three players, with the additional reseller, as shown in Figure 3. At the beginning of each time slot $t$, the client requests service from the reseller. The reseller in turn requests service from the access point, who replies her with a slot price $c_{t}$. Based on $c_{t}$, the reseller decides how to charge and sends a slot price $p_{t}$ to the client. The client chooses to accept or reject the price. If the client accepts $p_{t}$, the reseller replies "accept" to the access point; and vice versa. When game ends, resulted from the first rejection of a slot price by the client, the net payoff of the client is $F(T, \tau)-\sum_{t=1}^{T} p_{t}$, while the reseller and the access point have profit of $\sum_{t=1}^{T}\left(p_{t}-c_{t}\right)$ and $\sum_{t=1}^{T} c_{t}$ respectively. Authors in [10] prove that the following strategy profile is a PBE:

- The client follows the myopic strategy, connecting iff $t \leq \tau$ and $p_{t} \leq U$;

- The reseller picks a price mark-up function $p^{*}(c)$ that satisfies the properties:

$$
\begin{aligned}
& p^{*}(c) \in \arg \max _{p}(p-c) P(U \geq p) \\
& p^{*}\left(c^{\prime}\right) \geq p^{*}(c) \quad \forall c^{\prime}>c
\end{aligned}
$$

and charges the price $p_{t}=p^{*}\left(c_{t}\right)$ in slot $t$;

- The access point charges a non-decreasing price sequence $\left\{c_{t}\right\}$ such that

$$
c_{t} \in \arg \max _{c} c P\left(U \geq p^{*}(c)\right) .
$$

As in the one-hop case, it is common for the access point and the reseller to adopt a fixed-price strategy, since most distributions of $U$ yield single maximizers of $(p-c) P(U \geq$ $p)$ and $c P\left(U \geq p^{*}(c)\right)$ respectively.

The most important result of [10] is the proof of the natural selection of the fixed-rate pricing strategy by the access point and the reseller, without the need of contract enforcement. Fixed-rate pricing is appealing to customers for its simple charging scheme; while the exclusion of contract enforcement allows the service mechanism to be on a pure peer-to-peer basis and hence be scalable. However, as we are going to show in Section 4, this result only applies to the following special situations:

- The wireless network channel and the access point's uplink have an unlimited capacity, or have a sufficient capacity to meet all demands;

- The network has a limited capacity, but the access point does not provide bandwidth guarantee to clients; while clients' valuations of the service are independent to its quality.
It is obvious that the first condition is not always true, while the second condition may not be realistic. In networks where the above conditions do not hold, the pricing and purchasing strategies of nodes remain to be investigated.

\section{Extensions to the Unlimited Capacity Model-Multi-hop Case}

In this section, we extend the analysis under the unlimited capacity assumption into the multi-hop case, which is derived naturally from the two-hop case in [10]. The multihop case describes pricing dynamics in a multi-hop wireless network where a client is at an arbitrary number of hops away from the access point. We first define the model and some notations, then propose a game PBE. Results in this section also contribute to the solution to the multi-hop case under the limited capacity model later in Section 4.2.

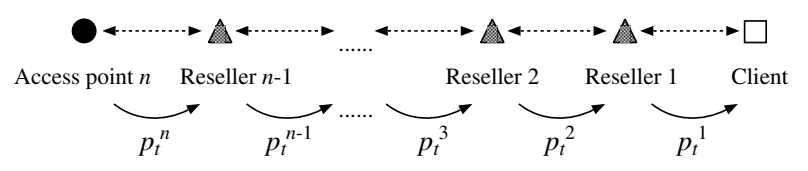

\section{Figure 4. Game modeling of the multi-hop case}

The multi-hop case allows clients to be arbitrarily $n$-hop away from the access point. Due to the unlimited capacity assumption, we can abstract the aggregated system to a game involving all nodes on the path from a client to the access point. The game involves $n+1$ players: the access point, $n-1$ resellers and the client, as shown in Figure 4. The resellers are indexed from the client side to the access point side by 1 to $n-1$, while the access point is indexed by $n$. Procedures for price negotiation are analogous to that in the two-hop case: at each time slot $t$, access point $n$ charges reseller $n-1$ a price $p_{t}^{n}$, who in turn charges reseller $n-2$ a price $p_{t}^{n-1}$ and so on; in the end, the client receives a price $p_{t}^{1}$ from reseller 1 . The net payoff of the client is $F(T, \tau)-\sum_{t=1}^{T} p_{t}^{1}$ for a usage of $T$ time slots. The net payoff for reseller $i$ is $\sum_{t=1}^{T}\left(p_{t}^{i}-p_{t}^{i+1}\right)$, for $i=1, \ldots, n-1$, and for the access point, the payoff is $\sum_{t=1}^{T} p_{t}^{n}$.

To determine the optimal pricing and a perfect Bayesian equilibrium for the unlimited capacity, multi-hop wireless network, we propose the following strategy profile:

\section{Strategy Profile for Multi-hop Wireless Mesh Network:}

1. The client follows the myopic strategy, connecting iff $t \leq \tau$ and $p_{t}^{1} \leq U$

2. Reseller $i$, for all $i \in\{1, \ldots, n-1\}$, picks a price mark-up function $p^{i *}\left(p^{i+1}\right)$ that satisfies the proper- 
ties:

$$
\begin{aligned}
& p^{i^{*}}\left(p^{i+1}\right) \in \arg \max _{p^{i}}\left[\left(p^{i}-p^{i+1}\right) P\left(U \geq m^{i}\left(p^{i}\right)\right)\right] \\
& p^{i^{*}}\left(p^{i+1^{\prime}}\right) \geq p^{i^{*}}\left(p^{i+1}\right) \quad \forall p^{i+1^{\prime}}>p^{i+1}
\end{aligned}
$$

and charges the price $p_{t}^{i}=p^{i *}\left(p_{t}^{i+1}\right)$ in time slot $t$;

3. Access point $n$ charges a non-decreasing price sequence $\left\{p_{t}^{n}\right\}$ with

$$
p_{t}^{n} \in \arg \max _{p^{n}}\left[p^{n} P\left(U \geq m^{n}\left(p^{n}\right)\right)\right]
$$

where the function $m^{i}\left(p^{i}\right)$ is defined for all $i \in\{1, \ldots, n\}$ as follows:

$m^{i}\left(p^{i}\right) \triangleq \begin{cases}p^{1^{*}}\left(p^{2^{*}}\left(\ldots\left(p^{i-1^{*}}\left(p^{i}\right)\right) \ldots\right)\right) \forall i \in\{2, \ldots, n\} \\ p^{1} & i=1 .\end{cases}$

The function $m^{i}\left(p^{i}\right)$ represents the price received by the client after the price $p^{i}$ set by node $i$ is marked up by all its downstream resellers.

It can be proven that the above strategy profile is indeed a PBE. The proof follows naturally from the proof of the twohop case PBE in [10]. The complete proof is obtainable in our technical report [7].

\section{Limited Capacity Model}

The formulation of the unlimited capacity model relies on the assumption that the wireless network channel and the access point's uplink have an unlimited capacity, or the access point provides no bandwidth guarantee to clients. In this section, we consider a more realistic scenario and inspect the economics in wireless networks in which there is a limited network capacity. Similar to the previous section, we begin with a one-hop network and show why the previous one-hop case PBE is not applicable under this new setting. A substitute for the access service provision model named "fixed-rate, non-interrupted service" is hence proposed and we provide an algorithm to obtain the optimal strategy of the access point in its defined strategy space. The analysis is finished with an extension to the multi-hop case.

\subsection{One-hop Case}

Here, we first present the necessary modifications to the original unlimited capacity model and transform it into the limited capacity version. The one-hop case of the limited capacity model still describes a wireless network consisting of an access point, plus clients who reach the access point directly. The distinction between the two models is that the wireless network and the access point's uplink here have a

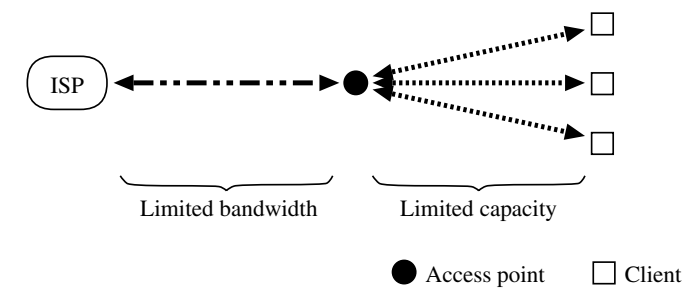

\section{Figure 5. Network diagram with channel ca- pacity for the one-hop case}

limited capacity, and the access point has to assure clients that they will have a certain amount of dedicated bandwidth. This imposes the access point a bandwidth constraint on its profit maximization problem. Figure 5 depicts this scenario. In our model, we limit the access point to admit at most $m>0$ clients at a time. Any client who arrives at the access point not being immediately served due to this limit will be dropped.

Another addition to the original model is an explicit client arrival process at the access point. This is necessary as the interactions between the access point and a client are now complicated by the removal of the unlimited capacity assumption - they cannot be summarized by one simple two-player game; the access point must decide its strategy on each occasion, based on its system condition at the time, such as the remaining capacity for admission. We model the client arrival behavior using a Poisson input process with a finite population of clients. Each client arrives with a rate $\lambda$ at the access point, and there is a total of $M$ clients in the population.

The last modification to the unlimited capacity model is to transform it from a discrete-slot process into a continuous-time process so as to ease our analysis when matched with the client arrival model. In the continuoustime version, the access point charges a particular client a price per unit time, or rate, $p(t)$ at time $t$. The variables $T$, $\tau$ and $U$ are converted, in the continuous-time sense, to represent the amount of time the client connects, the amount of time the client intends to connect, and the client's utility of the service per unit time respectively. The continuous-time web browsing utility function of the client thus remains the same as its previous form:

$$
F(T, \tau)=U \cdot \min (T, \tau) .
$$

The access point still only knows the probability distributions of $U$ and $\tau$. Here, we further assume that the access point takes $\tau$ to be exponentially distributed with mean $1 / \mu$. Our formulation of the limited capacity model is now complete. It should be clear to see the correspondence between our model and the classical M/M/m/m/M queuing system [6]. 
We follow by giving a simple scenario to show that under the limited capacity model, the access point, on some occasions, will choose either to charge clients with a variable rate, or to deliberately disconnect clients, rather than adopting a fixed-rate, non-interrupting strategy similar to the one in the one-hop case PBE of the unlimited capacity model proposed in [10].

Lemma 1 A fixed-rate, non-interrupting strategy is not at all time optimal to the access point under the limited capacity model.

Proof: Consider the scenario that a new client arrives at the access point, when it is at its full capacity. Let $p_{0}$ be the price of one of the $m$ connected clients is paying. The access point may announce a price $p_{0}{ }^{\prime}>p_{0}$ to the new client. If the new client accepts, the access point's best response is to disconnect the old client paying $p_{0}$ and admit the new one, unless the old client accepts a raise of price from $p_{0}$ to $p_{0}{ }^{\prime}$. Thus, a fixed-rate, non-interrupting strategy is not the best response of the access point at some time of the process.

Although the access point will wish to cease service to clients or increase the price over time to obtain higher profit, it is reasonable to believe that clients will be discouraged from buying such a kind of service since it is unrealistic to require clients to monitor the varying price continuously. Thus, we investigate a more practical service model, named the "fixed-rate, non-interrupted service".

The fixed-rate, non-interrupted service model requires a contract to be negotiated between the access point and a particular client as follows:

- The access point provides connection service to the client until the client voluntarily disconnects;

- The client pays a fixed rate $p$ for the service. The total payment is $p$ times the duration of the service.

Note that the access point is still allowed to announce different "fixed rates" (or prices) to different clients under this scheme, but once announced, this fixed rate cannot be changed during the course of service for a particular client.

A strategy of the access point involves setting the charging rate to clients who want to be connected. The access point can make her decision based on a single parameter, namely, the number of connected clients in the system. Adopting queuing system notations, the number of connected clients in the system is represented by the current "state". For the $\mathrm{M} / \mathrm{M} / \mathrm{m} / \mathrm{m} / \mathrm{M}$ queuing system, it has $m+1$ states, from state 0 to state $m$. At state $k$, for all $k \in\{0, \ldots, m-1\}$, the access point has to decide the rate $p_{k}$ to charge the next "to-be-admitted" client. No decision has to be made at state $m$ as the access point is at its full capacity. Thus, a policy of the access point is completely characterized by the price or rate vector $\vec{p}=\left(p_{0}, p_{1}, \ldots, p_{m-1}\right)$.

With the fixed-rate, non-interrupted service contract, we see that clients will play the following strategy to maximize her payoff: connect the access point iff $U \geq p$; disconnect from the access point at time $t=\tau$, with the assumption that clients with $U \geq p$, utility per unit time not less than charged rate, will not deliberately reject the first presented rate and wait until she receives a lower rate at a later time when the access point is less congested. Also, for clients rejecting the first presented rate, our Poisson client arrival process may not accurately model their possible behavior of re-probing the access rate afterward.

We now derive an expression of the expected profit per unit time, or the gain, of the access point in the long run as a function of the rate vector $\vec{p}$. The general equilibrium solution for birth-death queuing systems [6] is employed. Note that a transition from state $k$ to state $k+1$, for all $k \in\{0, \ldots, m-1\}$, requires not only an arrival of a client, but also her willingness to accept the charged price $p_{k}$, therefore, the "arrival rate" of our model is different from that of the conventional $\mathrm{M} / \mathrm{M} / \mathrm{m} / \mathrm{m} / \mathrm{M}$ queuing system by a factor of $P\left(U \geq p_{k}\right)$ for each state $k, k \in\{0, \ldots, m-1\}$. The transition rates of our model are:

$$
\begin{aligned}
& \lambda_{k}= \begin{cases}\lambda(M-k) P\left(U \geq p_{k}\right) & k<m \\
0 & \text { otherwise }\end{cases} \\
& \mu_{k}=k \mu \\
& k=1,2, \ldots, m \text {. }
\end{aligned}
$$

With $\pi_{k}$ denoting the limiting probability that the system is in state $k$, for all $k \in\{0, \ldots, m\}$, they are given by

$$
\pi_{k}=\frac{\left(\begin{array}{c}
M \\
k
\end{array}\right)\left(\frac{\lambda}{\mu}\right)^{k} \prod_{i=0}^{k-1} P\left(U \geq p_{i}\right)}{\sum_{j=0}^{m}\left(\left(\begin{array}{c}
M \\
j
\end{array}\right)\left(\frac{\lambda}{\mu}\right)^{j} \prod_{i=0}^{j-1} P\left(U \geq p_{i}\right)\right)},
$$

where empty product is unity by convention. For simplification, consider that the access point earns an expected profit of $p_{k} / \mu$ immediately when a client connects at state $k$. Hence, the gain of the access point is

$$
\begin{aligned}
& G(\vec{p}) \\
& =\sum_{k=0}^{m-1} \pi_{k} \lambda_{k}\left(\frac{p_{k}}{\mu}\right) \\
& =\frac{\sum_{k=0}^{m-1}\left(p_{k}(M-k)\left(\begin{array}{c}
M \\
k
\end{array}\right)\left(\frac{\lambda}{\mu}\right)^{k+1} \prod_{i=0}^{k} P\left(U \geq p_{i}\right)\right)}{\sum_{k=0}^{m}\left(\left(\begin{array}{c}
M \\
k
\end{array}\right)\left(\frac{\lambda}{\mu}\right)^{k} \prod_{i=0}^{k-1} P\left(U \geq p_{i}\right)\right)} .
\end{aligned}
$$


The optimal policy of the access point can be obtained by maximizing Eq. (2) over the rate vector $\vec{p}$. However, using classical optimization techniques to derive a closedform solution of the optimal policy requires solving simultaneous non-linear equations, which is exceedingly complicated. Instead, we use the policy-iteration method in the Markovian decision theory [4] to determine the proper pricing for the above optimization problem.

The Policy-iteration Method: The policy-iteration method involves an iteration cycle of two parts: the valuedetermination operation and the policy-improvement routine. It uses the notation $g$ to denote the gain of the system, and introduces a set of relative values $v_{k}$, for all $k \in\{0,1, \ldots, m\}$, which has the physical meaning of which $v_{i}-v_{j}$ is the increase in the gain caused by starting the system in state $i$ rather than in state $j$. The algorithm is started in the policy-improvement routine with all relative values $v_{k}$ set to 0 .

The Value-determination Operation The valuedetermination operation evaluates a policy $\vec{p}$ generated by the policy-improvement routine. It requires solving the following set of equations for all relative values $v_{k}$ and $g$ by setting $v_{m}$ to zero:

$$
\begin{gathered}
g=\lambda M P\left(U \geq p_{0}\right) v_{1}-\lambda M P\left(U \geq p_{0}\right) v_{0} \\
\quad+\lambda M P\left(U \geq p_{0}\right)\left(\frac{p_{0}}{\mu}\right) \\
g=\quad \lambda(M-k) P\left(U \geq p_{k}\right) v_{k+1} \\
\quad-\left(\lambda(M-k) P\left(U \geq p_{k}\right)+k \mu\right) v_{k}+k \mu v_{k-1} \\
+\lambda(M-k) P\left(U \geq p_{k}\right)\left(\frac{p_{k}}{\mu}\right) \\
k=1,2, \ldots, m-1 \\
g=\quad-m \mu v_{m}+m \mu v_{m-1} .
\end{gathered}
$$

With the solution, the algorithm goes into the policyimprovement routine.

The Policy-improvement Routine Based on the relative values $v_{k}$ obtained for the current policy $\vec{p}$ in the valuedetermination operation, the policy-improvement routine seeks to improve the current policy by considering alternatives in each state. It requires solving the following separable optimization problem where the design variables are $p_{0}, p_{1}, \ldots, p_{m-1}$ :

$$
\begin{array}{cl}
\max & \lambda M P\left(U \geq p_{0}\right) v_{1}-\lambda M P\left(U \geq p_{0}\right) v_{0} \\
& +\lambda M P\left(U \geq p_{0}\right)\left(\frac{p_{0}}{\mu}\right) \\
\max & \lambda(M-k) P\left(U \geq p_{k}\right) v_{k+1} \\
& -\left(\lambda(M-k) P\left(U \geq p_{k}\right)+k \mu\right) v_{k}+k \mu v_{k-1} \\
& +\lambda(M-k) P\left(U \geq p_{k}\right)\left(\frac{p_{k}}{\mu}\right) \\
k=1,2, \ldots, m-1 &
\end{array}
$$

When the client's utility rate $U$ has a uniform distribution on the interval $[a, b]$, one can derive a closed-form solution for the optimal rate $p_{k}{ }^{*}$ for each state $k$. As an optimal state rate $p_{k}{ }^{*}$ must lie on the interval $[a, b]$, we may substitute $P\left(U \geq p_{k}\right)$ with $\left(b-p_{k}\right) /(b-a)$. Differentiation followed by root finding yields the optimal $p_{k}{ }^{*}$ for each state $k$ :

$$
p_{k}^{*}=\frac{b-\mu\left(v_{k+1}-v_{k}\right)}{2} \quad k=0,1, \ldots, m-1 .
$$

The solution to the optimization problem then forms a new policy. If the difference between this new policy and the previous policy is smaller than a pre-defined threshold, the iteration process has converged and the (near-)optimal policy is found. Otherwise, the algorithm goes back to the value-determination operation and the new policy is evaluated.

The policy-iteration method reduces the profit maximization problem of the access point to solving sets of $m+1$ simultaneous linear equations in the value-determination operation, and sets of $m$ independent one-dimensional optimization problems in the policy-improvement routine. The computational complexity is reduced (as compared with the standard numerical optimization method), and it is shown in [4] that the above procedures guarantee the convergence to the best policy.

Here we show some numerical results obtained using the policy-iteration method. Cases in which client's utility rate $U$ is uniformly distributed on $[0,10]$, or normally distributed with a mean of 5 and a standard deviation of 1.67 are studied. We illustrate the state rates given by the policy-iteration method when the access point can support $m=5$ clients, and there are totally $M=10$ potential clients who want to receive the connection service. We fix the departure rate of client $\mu$ to 1 and vary the arrival rate $\lambda$ from 0.2 to 10 . Figure 6 shows the results. It can be observed that the statedependent price rises with the number of clients in the access point system: $p_{i} \geq p_{j}$, for all $i>j$. This agrees with the economic sense that when the remaining resource, or supply, of service decreases, the price increases. Also, the state-dependent price rises with increasing $\lambda$, and this is logical as the arrival rate $\lambda$ represents demand.

Lastly, we find in our experiments that the convergence rate of the policy-iteration method is around four for various problem size $m$, the number of state prices to be determined, from 1 to 100. A detailed evaluation is documented in our technical report [7]. The algorithm proves to be efficient in our access point profit maximization problem.

\subsection{Multi-hop Case}

We now extend the limited capacity model to the multihop case. We make the assumption that the bandwidth bottleneck is at the wireless channel one-hop around the access point, or at the access point's uplink to the Internet, where traffic from all clients in the wireless mesh network merges. Hence, any reseller who has purchased Internet access service from her upstream will have adequate bandwidth for 


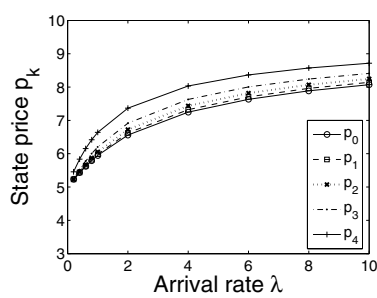

(a) $U$ uniformly distributed

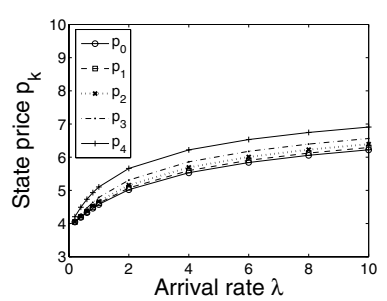

(b) $U$ normally distributed
Figure 6. State-dependent price with varying arrival rate

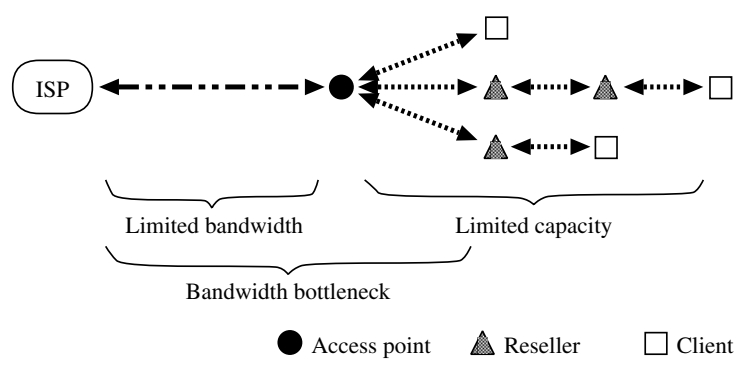

Figure 7. Network diagram with channel capacity for the multi-hop case

her downstream. This situation is depicted in Figure 7. In comparison with the multi-hop case in the unlimited capacity model, we see that the bandwidth constraint only affects the access point; for the resellers, their strategies only depend on the prices their respective upstream hops charge them. Thus, any node apart from the access point will follow her strategy in the unlimited capacity model here.

The focus of the multi-hop case is to devise the optimal pricing strategy of the access point, which involves determining the respective optimal prices for clients from different distances at each state. Thus, for an access point with capacity $m$, and with the assumption that the most distant clients arriving at the access point are from $n$ hops away, a policy of the access point can be characterized by the price matrix $\mathbf{P}$ consisting of elements $p_{k i}, k \in\{0, \ldots, m-1\}$, $i \in\{1, \ldots, n\}$, in which $p_{k i}$ represents the price at state $k$ for a client $i$-hop away.

To ease analysis, we modify the client arrival process by removing the feature of finite client population. This is necessary, as an arrival process with finite population requires keeping track of the numbers of admitted clients at different distances, which highly complicates state information. We roll back to an arrival model originated from the M/M/m/m queuing system [6]. Assuming the most distant clients ar- rive from a distance of $n$ hops, we use an arrival rate vector $\vec{\lambda}=\left(\lambda_{1}, \lambda_{2}, \ldots, \lambda_{n}\right)$ to denote the arrival rates of clients from different distances, in which $\lambda_{i}$ denotes the arrival rate of clients $i$-hop away. With $\mu$ denoting the departure rate of a client in the system, we have the following state transition rates:

$$
\begin{aligned}
& \lambda_{k}= \begin{cases}\sum_{i=1}^{n} \lambda_{i} P\left(U \geq m^{i}\left(p_{k i}\right)\right) & k<m \\
0 & \text { otherwise }\end{cases} \\
& \mu_{k}=k \mu \quad k=1,2, \ldots, m .
\end{aligned}
$$

The factor moderating $\lambda_{i}$, the pure arrival rate of clients $i$-hop away, is now $P\left(U \geq m^{i}\left(p_{k i}\right)\right)$, which is a simple reflection from the multi-hop case in the unlimited capacity model that any state rate $p_{k i}$ charged by the access point will be marked up by all $i-1$ downstream resellers, as expressed by the function $m^{i}\left(p_{k i}\right)$ in Eq. (1).

Further taking the simplification that the access point earns an expected profit of $p_{k i} / \mu$ immediately when a client $i$-hop away connects at state $k$, we can again use the policyiteration method to solve for the optimal pricing policy of the access point, but with the following changes. The set of equations to be solved in the value-determination operation is updated as:

$$
\begin{aligned}
g= & \left(\sum_{i=1}^{n} \lambda_{i} P\left(U \geq m^{i}\left(p_{0 i}\right)\right)\right) v_{1} \\
& -\left(\sum_{i=1}^{n} \lambda_{i} P\left(U \geq m^{i}\left(p_{0 i}\right)\right)\right) v_{0} \\
& +\sum_{i=1}^{n} \lambda_{i} P\left(U \geq m^{i}\left(p_{0 i}\right)\right)\left(\frac{p_{0 i}}{\mu}\right) \\
g= & \left(\sum_{i=1}^{n} \lambda_{i} P\left(U \geq m^{i}\left(p_{k i}\right)\right)\right) v_{k+1} \\
& -\left(\sum_{i=1}^{n} \lambda_{i} P\left(U \geq m^{i}\left(p_{k i}\right)\right)+k \mu\right) v_{k} \\
& +k \mu v_{k-1}+\sum_{i=1}^{n} \lambda_{i} P\left(U \geq m^{i}\left(p_{k i}\right)\right)\left(\frac{p_{k i}}{\mu}\right) \\
g= & -m \mu v_{m}+m \mu v_{m-1} .
\end{aligned}
$$

The set of optimization problems in the policyimprovement routine is updated as:

$$
\begin{array}{cl}
\max & \left(\sum_{i=1}^{n} \lambda_{i} P\left(U \geq m^{i}\left(p_{0 i}\right)\right)\right) v_{1} \\
& -\left(\sum_{i=1}^{n} \lambda_{i} P\left(U \geq m^{i}\left(p_{0 i}\right)\right)\right) v_{0} \\
& +\sum_{i=1}^{n} \lambda_{i} P\left(U \geq m^{i}\left(p_{0 i}\right)\right)\left(\frac{p_{0 i}}{\mu}\right) \\
\max \quad\left(\sum_{i=1}^{n} \lambda_{i} P\left(U \geq m^{i}\left(p_{k i}\right)\right)\right) v_{k+1} \\
\quad-\left(\sum_{i=1}^{n} \lambda_{i} P\left(U \geq m^{i}\left(p_{k i}\right)\right)+k \mu\right) v_{k} \\
\quad+k \mu v_{k-1}+\sum_{i=1}^{n} \lambda_{i} P\left(U \geq m^{i}\left(p_{k i}\right)\right)\left(\frac{p_{k i}}{\mu}\right) \\
k=1,2, \ldots, m-1,
\end{array}
$$




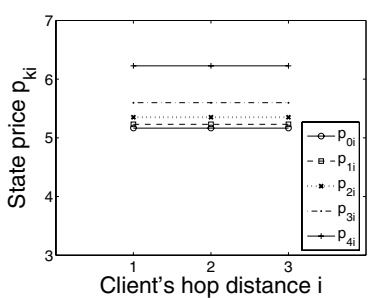

(a) $U$ uniformly distributed

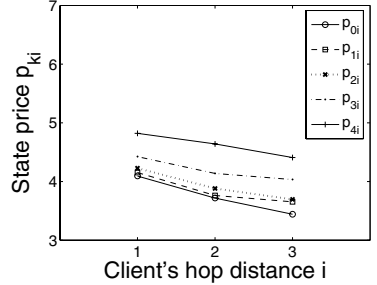

(b) $U$ normally distributed

\section{Figure 8. Price matrix with different utility rate distributions}

where the design variables are now $p_{k i}, k \in\{0, \ldots, m-1\}$, $i \in\{1, \ldots, n\}$. When client's utility rate $U$ has a uniform distribution on the interval $[a, b]$, we again has a closed-form solution for the optimal rate $p_{k i}{ }^{*}$ for clients $i$-hop away for each state $k$ :

$$
\begin{aligned}
& p_{k i}{ }^{*}=\frac{b-\mu\left(v_{k+1}-v_{k}\right)}{2} \\
& k=0,1, \ldots, m-1 \text { and } i=1,2, \ldots, n \text {. }
\end{aligned}
$$

It can be observed that, when $U$ has a uniform distribution, optimal prices for clients at different distances are the same for each state.

The following shows some numerical results of the multi-hop case obtained by using the policy-iteration method. Figure 8 essentially plots the resulting optimal price matrices for two cases. The first case has the client's utility rate $U$ uniformly distributed on $[0,10]$; the second case has $U$ normally distributed with a mean of 5 and a standard deviation of 1.67. For both cases, the arrival rate vector $\vec{\lambda}$ is $(4,2,1)$, the departure rate $\mu$ is 1 , and the capacity of the access point $m$ is 5 . It can be verified that when $U$ is uniformly distributed, the optimal prices for clients at different distances at each state are identical; while when $U$ is normally distributed, the access point tends to charge a lower price for clients further away. As in the one-hop case, prices rise with the number of admitted clients.

\section{Conclusion}

We have conducted a mathematical analysis of the economic behavior of nodes in a wireless mesh network, when they are making decision to establish an Internet connection service. Two scenarios are investigated: either the network has an unlimited or limited channel capacity. For the unlimited capacity case, we extend the analysis in [10] to cover the multi-hop scenario. In the limited capacity case, we have proved that a fixed-rate pricing scheme similar to the one proposed in [10] is not optimal, or economically beneficial, to the access point. We further investigate a more practical "fixed-rate, non-interrupted service" model for charging. To determine the optimal price for this charging scheme, we model the problem as a Markovian decision process and use the efficient policy-iteration method to solve for the optimal pricing strategy of the access point. Numerical results show that the state price follows with supply and demand.

\section{Acknowledgment}

The work of D. M. Chiu is supported in part by RGC grant 411505 . The work of J.C.S. Lui is supported in part by the RGC grant. This work is affiliated with the MicrosoftCUHK Joint Laboratory for Human-centric Computing and Interface Technologies.

\section{References}

[1] K. Chen, Z. Yang, C. Wagener, and K. Nahrstedt. Market models and pricing mechanisms in a multihop wireless hotspot network. In Proc. of ACM MobiQuitous Conference, July 2005.

[2] E. Friedman and D. Parkes. Pricing WiFi at Starbucks issues in online mechanism design. In Proc. Fourth ACM Conf. on Elec. Commerce (EC'03), 2003.

[3] D. Fudenberg and J. Tirole. Game Theory, chapter 8. MIT Press, 1991.

[4] R. A. Howard. Dynamic Programming and Markov Processes, chapter 8. MIT Press and John Wiley \& Sons, 1960.

[5] F. Kelly, A. Maulloo, and D. Tan. Rate control in communication networks: shadow prices, proportional fairness and stability. In Journal of the Operational Research Society, volume 49, 1998.

[6] L. Kleinrock. Queueing systems, chapter 3. John Wiley \& Sons, 1975.

[7] R. K. Lam, D.-M. Chiu, and J. C. S. Lui. On the access pricing issues of wireless mesh networks. Technical report, The Chinese Univ. of Hong Kong.

[8] J. K. MacKie-Mason and H. R. Varian. Pricing the Internet In Public Access to the Internet, JFK School of Government, page 37, May 26-27, 1993.

[9] S. Micali and R. L. Rivest. Micropayments revisited. In CT-RSA, pages 149-163, 2002.

[10] J. Musacchio and J. Walrand. WiFi access point pricing as a dynamic game. IEEE/ACM Trans. Networking. to appear in

[11] R. L. Rivest and A. Shamir. Payword and micromint: Two simple micropayment schemes. In Security Protocols Workshop, pages 69-87, 1996.

[12] E. Viterbo and C. Chiasserini. Dynamic pricing in wireless networks. In International Symposium on Telecommunications, pages 385-388, Sept. 2001 